\title{
Signature of anisotropic exchange interaction revealed by vector-field control of the helical order in a FeGe thin plate
}

\author{
Victor Ukleev $\odot,{ }^{1, *}$ Oleg Utesov $\odot,{ }^{2,3,4}$ Le Yu,${ }^{1,5,6}$ Chen Luo $\odot,{ }^{7}$ Kai Chen, ${ }^{7}$ Florin Radu $\odot,{ }^{7}$ Yuichi Yamasaki, ${ }^{8,9}$ \\ Naoya Kanazawa, ${ }^{10}$ Yoshinori Tokura, ${ }^{10,11,12}$ Taka-hisa Arima,,${ }^{11,13}$ and Jonathan S. White ${ }^{1}$ \\ ${ }^{1}$ Laboratory for Neutron Scattering and Imaging (LNS), Paul Scherrer Institute (PSI), CH-5232 Villigen, Switzerland \\ ${ }^{2}$ Petersburg Nuclear Physics Institute NRC “Kurchatov Institute”, Gatchina, Saint-Petersburg 188300, Russia \\ ${ }^{3}$ St. Petersburg State University, 7/9 Universitetskaya nab., St. Petersburg 199034, Russia \\ ${ }^{4}$ National Research University Higher School of Economics, Soyuza Pechatnikov, 16, St. Petersburg, 190008, Russia \\ ${ }^{5}$ Laboratory for Ultrafast Microscopy and Electron Scattering (LUMES), Institute of Physics, École Polytechnique Fédérale de Lausanne \\ (EPFL), CH-1015 Lausanne, Switzerland \\ ${ }^{6}$ Laboratory of Nanoscale Magnetic Materials and Magnonics (LMGN), Institute of Materials, École Polytechnique Fédérale de Lausanne \\ (EPFL), CH-1015 Lausanne, Switzerland \\ ${ }^{7}$ Helmholtz-Zentrum Berlin for Materials and Energy, Albert Einstein Str. 15, 12489 Berlin, Germany \\ ${ }^{8}$ Research and Services Division of Materials Data and Integrated System (MaDIS), National Institute \\ for Materials Science (NIMS), Tsukuba, 305-0047 Japan \\ ${ }^{9}$ PRESTO, Japan Science and Technology Agency (JST), Kawaguchi 332-0012, Japan \\ ${ }^{10}$ Department of Applied Physics, University of Tokyo, Tokyo 113-8656, Japan \\ ${ }^{11}$ RIKEN Center for Emergent Matter Science (CEMS), Wako 351-0198, Japan \\ ${ }^{12}$ Tokyo College, University of Tokyo, Tokyo 113-8656, Japan \\ ${ }^{13}$ Department of Advanced Materials Science, University of Tokyo, Kashiwa 277-8561, Japan
}

(Received 2 November 2020; revised 13 December 2020; accepted 24 December 2020; published 29 January 2021)

\begin{abstract}
We investigated the low-temperature helical magnetic structures in a thin plate of chiral magnet FeGe, paying special attention to the variation of the magnetic modulation period with the angle of an applied magnetic field. A unique vector-field setup allowed us to continuously rotate the helical texture in the sample plane while measuring high-resolution resonant small-angle x-ray scattering patterns. The experiments and supporting micromagnetic simulations show that the direction of helical propagation can be robustly controlled by directional magnetic field training. The observed anisotropy of the helical modulation period is explained naturally by inclusion of higher-order anisotropic exchange in the Bak-Jensen model. The observation and estimation of this interaction provides a pathway to refine theoretical models of cubic chiral magnets and design spintronics devices based on the propagation switching of helical textures.
\end{abstract}

DOI: 10.1103/PhysRevResearch.3.013094

\section{INTRODUCTION}

Magnetic skyrmions are topological spin vortices in chiral and polar magnetic materials that attract significant interest due to their promising properties for magnetic storage and other spintronic applications [1-3]. Noncentrosymmetric B20-type $\mathrm{FeGe}, \mathrm{MnSi}, \mathrm{Fe}_{1-x} \mathrm{Co}_{x} \mathrm{Si}$, and related structures are prototypical chiral cubic magnets that host skyrmions [1,4,5], and the control of magnetic states in these materials is important from both fundamental and applied research perspectives. For example, it has been shown recently that various magnetic textures in thin layers of the near room temperature helimagnet $\mathrm{FeGe}$ can be efficiently created and manipulated

\footnotetext{
*victor.ukleev@psi.ch

Published by the American Physical Society under the terms of the Creative Commons Attribution 4.0 International license. Further distribution of this work must maintain attribution to the author(s) and the published article's title, journal citation, and DOI.
}

by external magnetic fields [6-8], microwave radiation [9], electric currents [10-12], light pulses [13,14], and mechanical deformations [15-18].

The ground-state magnetic structures of B20-type systems are well explained by the Bak-Jensen model that takes into account the interplay between Heisenberg exchange interaction, antisymmetric Dzyaloshinskii-Moriya interaction (DMI) $[19,20]$, anisotropic exchange interaction (AEI), and cubic anisotropy [21]. The weak cubic anisotropy determines the spin-wave gap and some additional peculiarities of the helix axis orientation under a magnetic field, while the exchange, DMI, and AEI are responsible for the helical magnetic structure and its orientation relative to crystallographic axes [22]. The latter interaction is often neglected due to its weak impact on experimental observations, and the commonly used definition of the helical wavevector $Q$ is given by $Q=S D / A_{e x}$, where $S$ is the spin, $D$ is the DMI constant, and $A_{e x}$ is the exchange stiffness [22]. However, the cubic and exchange anisotropies are important for determining the propagation direction of the magnetic helix. For a fixed sign of the cubic anisotropy constant $K_{c}>0$, helices propagate along [100]- 
equivalent axes in the case of a positive AEI constant $F>0$ (for example $\mathrm{Cu}_{2} \mathrm{OSeO}_{3}, \mathrm{Fe}_{0.85} \mathrm{Co}_{0.15} \mathrm{Si}$ ) and $\hat{Q} \|[111]$ if $F<$ 0 (MnSi). Interestingly, in FeGe the reorientation of the spiral propagation vector from high- $T \hat{Q} \|[100]$ to low- $T \hat{Q} \|[111]$ is observed in FeGe at $T \approx 220 \mathrm{~K}$ [23], indicating a sign change of $F$ from positive to negative values on cooling. This transition displays a temperature hysteresis of the order of $20 \mathrm{~K}$ within which the two orientations of the propagation vector may coexist, indicating that the $\mathrm{AE}$ fluctuates around zero. Similar effects were demonstrated for $\mathrm{Mn}_{1-x} \mathrm{Fe}_{x} \mathrm{Si}$ and $\mathrm{Fe}_{1-x} \mathrm{Co}_{x} \mathrm{Si}$ solution series [24,25].

Typically, the AEI constant is estimated experimentally from the helical-to-conical transition field $B_{c 1}$ as $F=$ $2 S g \mu_{B} B_{c 1} / Q^{2}$, where $g$ is the Landé factor and $\mu_{B}$ is the Bohr magneton. This formula leads to the estimated low-temperature value of $F \approx-45 \mathrm{meV} \AA^{2}$ for FeGe [26]. However, direct demonstration of the influence of the exchange anisotropy on the magnitude of the $Q$ vector has not been reported for any of the B20-type compounds except for prototype $\mathrm{MnSi}[27,28]$. Theoretically it has been shown that the AEI can induce shape deformation of a skyrmion and coordination of the skyrmion lattice in a chiral magnet [29]. Therefore, an independent demonstration of the effect of the AEI on magnetic periodicity and determination of the AEI constant are important for the theoretical understanding of the magnetic properties of FeGe on the microscopic scale [30], and for further improvement for the modeling accuracy of topological spin textures in FeGe-based nanostructures and spintronics devices [16,31-33]. Moreover, the interplay between cubic anisotropy, AEI, and magnetic field has been proposed to be crucial for the stability of the recently discovered tilted conical and low-temperature skyrmion states in $\mathrm{Cu}_{2} \mathrm{OSeO}_{3}$ [34-36].

Recently, resonant elastic soft small-angle x-ray scattering (REXS) became an ultimate high-resolution probe to study long-periodic magnetic structures in chiral helimagnets [17,37-44]. In the present work we demonstrate the anisotropic character of the helical state in FeGe thin plate directly by means of high-resolution REXS. While the direction of the zero-field helical propagation vector $Q$ in a thin plate is remarkably flexible and can be arbitrarily aligned in the (110) plane by in-plane field training, we find that the magnitude of $Q$ nonetheless remains sensitive to its orientation in this plane. We argue that the observed in-plane variation in $Q$ of order $1 \%$ is determined by the higher order AEI in accord with the expectation of the Bak-Jensen model.

\section{EXPERIMENTAL}

The single-crystal sample of FeGe was grown by the chemical vapor transport method [45]. A lamella with a thickness of 150-200 nm was prepared by focused ion beam (FIB) milling, and placed behind an aperture of diameter $8 \mu \mathrm{m}$ on a goldcoated $\mathrm{Si}_{3} \mathrm{~N}_{4}$ membrane. The same FeGe thin plate sample was studied previously in Ref. [17]. The details of the lamella preparation and FIB milling routine can be found elsewhere $[38,39,46]$. Note that, at low temperatures, a tensile strain in the FeGe sample onsets on cooling due to the firm tungsten contacts on both sides of the thin plate and the difference between the thermal expansion coefficients of FeGe and the $\mathrm{Si}_{3} \mathrm{~N}_{4}$ membrane $[15,17,47,48]$.

The REXS experiment was carried out at the soft X-ray beamline PM2-VEKMAG [49] (BESSY-II, HelmholtzZentrum Berlin for Materials and Energy, Germany) during the multibunch operational mode of the synchrotron. The sample was placed in the high-vacuum chamber with a background pressure of $10^{-10}$ Torr, and in the gap of a vector-field superconducting magnet. REXS intensities were collected by a custom designed in-vacuum Peltier-cooled charge coupled device (CCD) detector manufactured by GreatEyes (Berlin, Germany) of $2048 \times 2048$ pixels. The CCD was protected from the direct beam by a tungsten beamstop with a size of $0.3 \mathrm{~mm}$. In the present experimental geometry the detector covered a scattering angle from $0.09^{\circ}$ to $2.1^{\circ}$. The exposure time for each REXS pattern was $120 \mathrm{~s}$.

X-ray absorption (XAS) and magnetic circular dichroism (XMCD) spectra were collected in transmission mode using a photodiode detector. The VEKMAG endstation provides magnetic fields up to $9 \mathrm{~T}$ in the beam direction and a $1 \mathrm{~T}$ vectorial field in all directions. This unique installation allowed us to rotate the magnetic field $\mathbf{B}(\theta)$ azimuthally in the sample plane, where $\theta=0^{\circ}$ corresponds to a horizontal field applied perpendicular to the beam axis. The sample temperature was controlled by an He flow-type refrigerator over a range from $2 \mathrm{~K}$ to $500 \mathrm{~K}$. Experiments were carried out in transmission geometry using circularly polarized soft x-rays. The XMCD spectrum was measured with a fixed photon helicity of the $\mathrm{X}$-ray beam by switching the magnetic field from $+1 \mathrm{~T}$ to $-1 \mathrm{~T}$. A maximum of the dichroic signal was found at photon energy $E=706 \mathrm{eV}$ corresponding to the Fe $L_{3}$ edge [Fig. 1(a)]. The observed transmission XMCD spectrum features, such as unbalanced $L_{3}$ and $L_{2}$ peak intensities and shapes, are affected strongly by the strong self-absorption of the sample. To additionally confirm the magnetic origin of the signal, the XMCD hysteresis loop was measured in the field parallel to the beam geometry [Fig. 1(b)]. The critical out-of-plane field value $B_{c 2} \approx 0.6 \mathrm{~T}$ for the conical-to-induced ferromagnetic transition can be deduced.

The position of the attenuated transmitted beam on the CCD camera was measured without the beamstop to precisely determine the reciprocal space origin. Due to the limitations of the experimental geometry-namely the fixed position of the $\mathrm{CCD}$ detector, and the fixed resonant $\mathrm{x}$-ray wavelength tuned to the $L_{3}$ edge-only one helical Bragg peak at $Q \approx 0.09 \mathrm{~nm}^{-1}$ was available in the detector range. The azimuthal acceptance of the CCD camera $\theta$ covered the range from $25^{\circ}$ to $115^{\circ}$, which included the principal crystallographic directions [111] and [110].

The sample was zero-field cooled from the room temperature to $T=2 \mathrm{~K}$. To study the zero-field helical wavevector as a function of the in-plane angle $|\mathbf{Q}(\theta)|$, saturating magnetic fields $B(\theta)=0.5 \mathrm{~T}$ were applied in the corresponding in-plane direction and consequently removed. Magnetic saturation of the sample at $0.5 \mathrm{~T}$ was ensured by in situ REXS, showing the absence of conical reflections for all measured $\theta$ angles. In the present experimental geometry the crystal was oriented in a way that the field $B_{\theta=0^{\circ}} \|[001]$, and the field $B_{\theta=90^{\circ}} \|[110]$. The fields were released from saturation to zero in $\Delta B=-0.25 \mathrm{~T}$ steps. At certain values of $\theta$, subsequent 

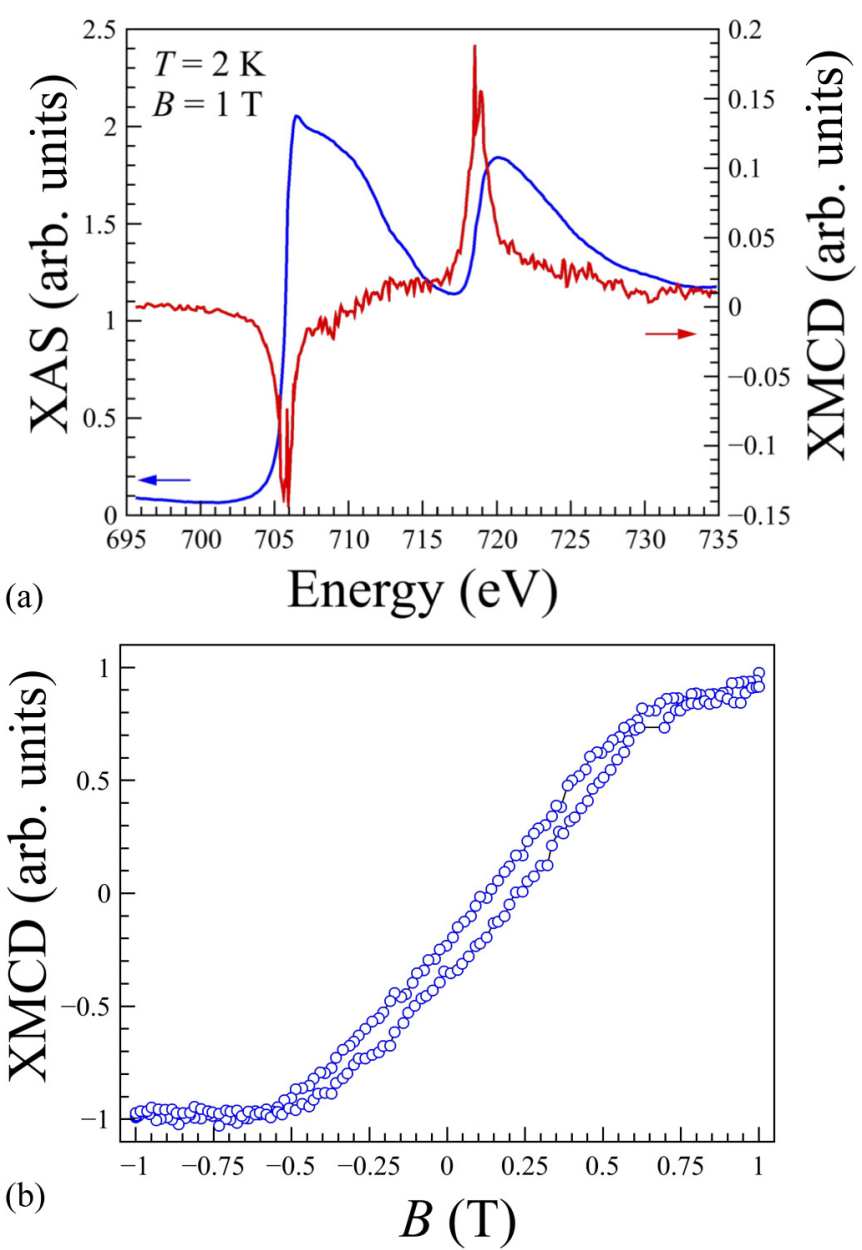

FIG. 1. (a) XAS and XMCD spectra recorded at $T=2 \mathrm{~K}$ and applied out-of-plane magnetic field $B=1 \mathrm{~T}$ in transmission mode. (b) The hysteresis XMCD loop measured at $T=2 \mathrm{~K}$ as a function of the out-of-plane magnetic field.

data were obtained under an increasing out-of-plane magnetic field to study the helical-to-conical transition field $B_{c 1}$.

\section{RESULTS AND DISCUSSION}

Figure 2(a) shows that the field training procedure described in the previous section results in a robust alignment of the helical wavevector along the direction of the applied in-plane field. A polar plot of the REXS intensity averaged over all zero-field scans as a function of applied field angle $\theta$ is shown in Fig. 2(b), along with the $Q$ dependence suggested by theory introduced later. Importantly, these metastable helical configurations allow us to investigate anisotropic properties of the crystal, similar to chiral fluctuations that allowed the observation of weakly anisotropic magnetic modulations in the paramagnetic phase of MnSi [27].

To reproduce numerically the directional training of helical order in a FeGe thin plate we performed micromagnetic simulations using MuMax $^{3}$ package [50]. In the simulation we assumed a two-dimensional plate of $1024 \times 1024$ cells with the in-plane cell size of $2 \mathrm{~nm}$, thickness of $150 \mathrm{~nm}$, and periodic boundary conditions along in-plane directions. Experimentally-determined values of the exchange stiffness $A_{e x}=8.78 \mathrm{pJ} / \mathrm{m}$ DMI constant $D=1.58 \mathrm{~mJ} / \mathrm{m}^{2}$, cubic anisotropy constant $K_{c 1}=10 \mathrm{~kJ} / \mathrm{m}^{3}$, and saturation magnetization $M_{s}=384 \mathrm{kA} / \mathrm{m}$ were taken from the microwave spin-wave spectroscopy [51].

In the present simulation, saturating in-plane magnetic fields $B(\theta)$ were initially applied to a random spin pattern and decreased to zero in steps following the experimental protocol, i.e., going from the field-polarized to helical state via a presumed intermediate in-plane conical state. The outof-plane magnetization component of the resulting zero-field helical order is shown in Fig. 2(c), with the corresponding fast Fourier transform (FFT) images shown in Fig. 2(d). The simulation result suggests that the helical propagation vector can be effectively aligned with any in-plane angle by a corresponding in-plane field training; this is consistent with our observations. The finite width of helical Bragg peaks in the $\theta$ plane of both the FFT images and the measured REXS patterns arises due to topological dislocations (merons and antimerons) at helical domain boundaries [52]. In the present work we do not consider the more complex case of three-dimensional magnetic structures that may emerge due to the finite-size effects in the thin plate of a chiral magnet [53-55].

Next, we discuss the variation in magnitude of the helical propagation vector $|\mathbf{Q}(\theta)|$, which is revealed by REXS to depend on the direction of the in-plane training field [Figs. 2(b) and 3]. The oscillation of $|Q|$ with a periodicity of $45^{\circ}$ [Figs. 2(b) and 3] could not be reproduced in the micromagnetic model that includes only the exchange interaction, DMI, and cubic anisotropy. This remains the case even while the demagnetization factor in the present twodimensional micromagnetic simulation might not be captured accurately. This aspect was explored through similar simulations with $2 \mathrm{~nm}$-thick cells and the rectangular shape of the sample, neither of which affected the result of the simulation. Therefore, we conclude that the demagnetization factor in the micromagnetic simulation does not affect the anisotropy of the spiral period in FeGe captured in the experiment. To thus explain the experimental observation, we consider the another anisotropic exchange interaction that is hitherto absent from standard micromagnetic simulation packages.

We thus address this issue analytically, and start by revisiting the basic properties of the Bak-Jensen model in the context of the anisotropic exchange interaction. On the level of a classical Kaplan helix energy, this interaction directly affects the spiral propagation vector of the system $[21,22]$. The classical energy per one spin of the planar spiral propagating along $\hat{c}$ direction is following:

$$
E=-\frac{S^{2} J_{\mathbf{Q}}}{2}-S^{2} D Q+\frac{S^{2} F Q^{2}}{4} I(\hat{c}),
$$

where $J_{\mathbf{Q}}=J_{\mathbf{0}}-A_{e x} Q^{2} / S\left(J_{\mathbf{Q}}\right.$ being Fourier transform of the exchange interaction), $I(\hat{c})=\sum_{v=x, y, z} c_{v}^{2}\left(a_{v}^{2}+b_{v}^{2}\right)$ is the cubic invariant ( $\hat{a}, \hat{b}$ are basis vectors of the spiral plane), see Ref. [22]. So, the spiral vector reads as

$$
\mathbf{Q}=\frac{S D \hat{c}}{A_{e x}+S F I(\hat{c}) / 2} .
$$



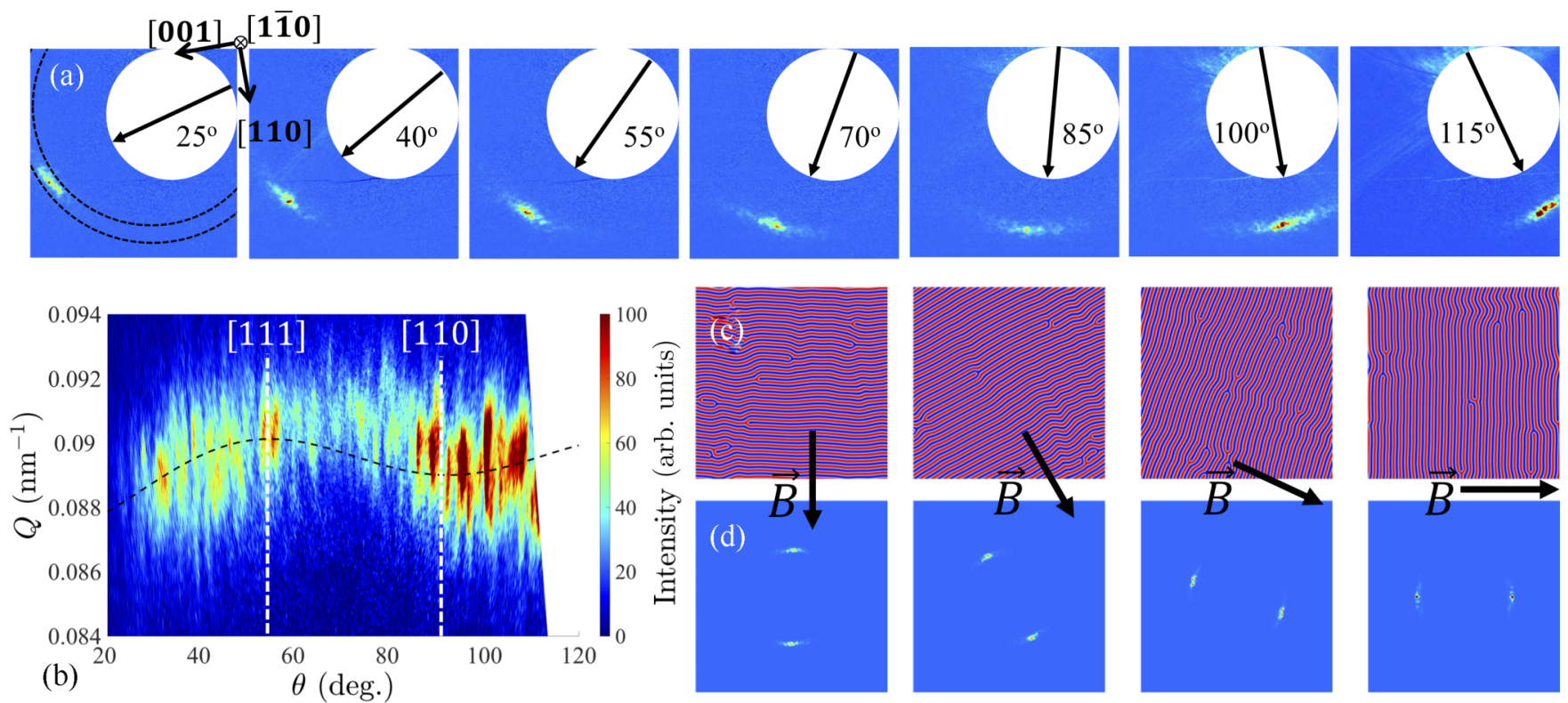

FIG. 2. (a) Zero-field REXS patterns recorded after the in-plane field training procedure at an angle $\theta$. Dashed lines indicate the data integration region used for constructing panel (b). Arrows show magnetic field orientation respectively to crystallographic axes. (b) Polar plot of the REXS intensity pattern averaged over all measured zero-field states after a corresponding field training at $\theta=25^{\circ} \ldots 115^{\circ}$ with $5^{\circ}$ step. The dashed black line indicates the fitted $Q$ dependence as discussed in the main text. Coordinates $\theta$ of crystallographic [111] and [110] axes of the sample are indicated as white dashed lines. (c) Real-space and (d) FFT images of the out-of-plane magnetization component derived from micromagnetic simulations of zero-field helical textures aligned by the field-training procedure. The directions of the corresponding training fields are shown by black arrows.

Due to the smallness of the anisotropic exchange constant $F$, we can write the spiral vector in the form:

$$
\mathbf{Q}=\frac{S D \hat{c}}{A_{e x}}\left(1-\frac{S F I(\hat{c})}{2 A_{e x}}\right)
$$

which shows that the small correction to the "bare" spiral vector value $S D / A_{e x}$ is governed by the cubic invariant. Its maximal value is $2 / 3$ for cubic diagonals $(\mathbf{Q} \|[111])$, and its minimum is 0 for cubic edges $(\mathbf{Q} \|[100])$. Using Eq. (1) we

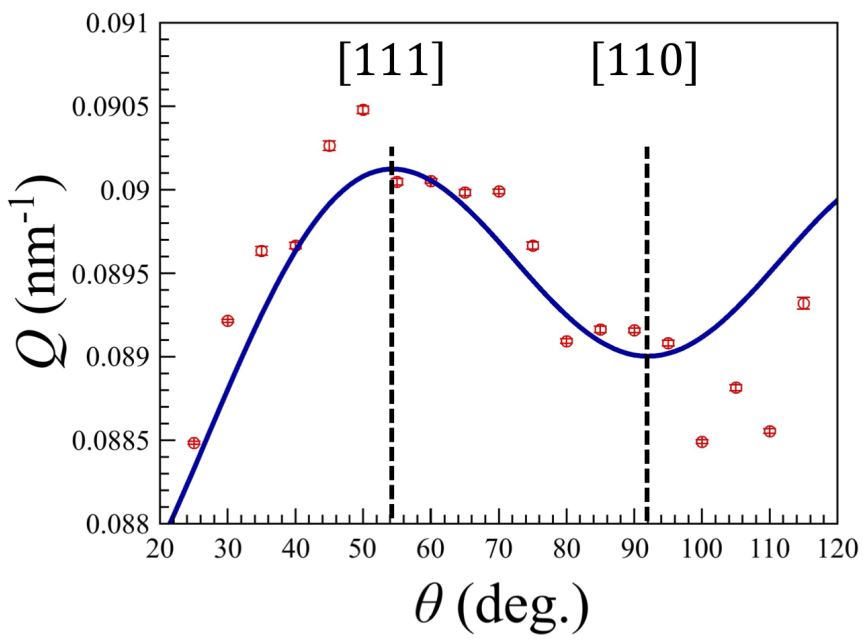

FIG. 3. Zero-field $Q$ magnitude measured as a function of the inplane angle $\theta$ after corresponding field training. The solid line shows the fitted curve according to the Eq. (3). obtain

$$
E=-\frac{S^{2} J_{0}}{2}-\frac{S^{2} D Q}{2} .
$$

For $\mathrm{FeGe}$ in equilibrium at low temperature, the spiral vector is oriented along the cubic diagonal, thus it is maximal when aligned with [111] and so $F<0$.

In the present paper we are especially interested in the plane defined by the basis vectors [110] and [001]. Therefore, in a conventional spherical coordinate system we have $\varphi=45^{\circ}$, and

$$
I(\hat{c})=I(\theta)=\frac{\sin ^{2} \theta\left(1+3 \cos ^{2} \theta\right)}{2} .
$$

We plug this function into Eq. (3) and find that it describes the experimental data well (see Fig. 3).

An important point to note here is that a tensile strain does not lead to any significant corrections to the predicted angle dependence of the spiral vector given by Eq. (3). A possible explanation for this can be that all the constants (exchange coupling, DMI, and AEI) are proportional to the integral describing electron wave-function overlap. This integral will vary with tensile strain, but will leave the ratios between parameters unchanged, and thus $\mathbf{Q}$ in Eq. (3) intact. However, to rule out completely any possible effects of tensile strain, data should be obtained for the full range of $\theta$ from 0 to $360^{\circ}$, and the symmetry of the measured curve analyzed.

Figure 3 shows a fit of the experimentally measured angle dependence of $|\mathbf{Q}(\theta)|$ using Eq. (3), which takes the AEI term into account. Using the exchange stiffness parameter of 
FeGe one obtains fitted values of $Q_{0}=S D / A_{e x}=0.0870 \pm$ $0.0011 \mathrm{~nm}^{-1}$ and an AEI constant $F=-39.5 \pm 17.9 \mathrm{meV}^{2}$ at $T=2 \mathrm{~K}$, which respectively agree with bulk neutron scattering data and the AEI constant estimation based on the value of $B_{c 1}[23,26]$. The negative sign of the exchange anisotropy constant $F$ is consistent with the preferred [111]-orientation of the spiral wavevector at low temperatures [23]. According to the cubic invariant [Eq. (5)], $|\mathbf{Q}(\theta)|$ reaches a maximum for the angle corresponding to the [111] direction, a minimum for the [100]-equivalent axes, and a local minimum for the [110] direction. These features are in good agreement with experimental data (Fig. 3). The deviation between the expected theoretical upturn of $|\mathbf{Q}(\theta)|$ for $\theta>100^{\circ}$ and the experimental data might be induced by the tensile strain effect, or a thickness gradient in the lamella sample $[17,56]$. The deviation, as well as the REXS intensity variation as a function of $\theta$ (Fig. 2(b), can be also related to a possible small (few degrees) offset of the sample that may result in the helical plane tilt towards the out-of-plane magnetic field training axis.

The presented framework should be generally applicable to the cubic chiral magnets, and can be further employed to explain the helical and skyrmion wavevector anisotropy already observed in $\mathrm{Fe}_{0.85} \mathrm{Co}_{0.15} \mathrm{Si}$ [57].

Next, we discuss the helix (meta)stability after the field training. Experimentally, after passing through the in-plane field-induced conical phase, the system relaxes to a helical phase that maintains the conical orientation of $\mathbf{Q}$, rather than $\mathbf{Q}$ realigned to some energetically favorable direction. A similar result is reproduced in the micromagnetic simulation presented earlier. This peculiar effect can be attributed to the metastability of the corresponding helices. Here we briefly discuss the origin of this behavior.

Let's consider a certain spin $\mathbf{S}_{0}$ and denote its alignment as parallel to an $x$ axis (so its phase is put to be zero). In a cubic helimagnet, and in the absence of magnetic field, there are pairs of neighboring spins with phases $\pm \phi$ which are $\mathbf{Q}$ dependent. Next, one can calculate an effective molecular field acting on the chosen spin from a single pair of neighbors and obtain

$$
\begin{aligned}
h_{x} & =J S[\cos \phi+\cos (-\phi)]+D S[\sin \phi-\sin (-\phi)], \\
h_{y} & =J S[\sin \phi+\sin (-\phi))+D S[\cos \phi-\cos (-\phi)] .
\end{aligned}
$$

The last quantity is zero for every planar helix no matter what the modulation vector $\mathbf{Q}$ is. Then, the molecular field acting on each spin is longitudinal and is much larger than the one from small anisotropic interactions. The latter, in comparison with the external magnetic field, does not provide a torque for the spiral as a whole, and will not reorient the spiral propagation vector $\mathbf{Q}$ chosen by the in-plane magnetic field. Thus, the whole spin texture is at least metastable. Anisotropy will lead only to an additional modulation of the metastable helix that results in higher harmonics with small amplitudes (see, e.g., Refs. [58,59]).

This simple picture should only be correct, however, if surface effects are negligible. It is seen from Eq. (6) that in this case the symmetry with respect to neighbors is violated for the surface spins. Nevertheless, in thin films with an in-plane helical propagation vector, the initially field-polarized spins
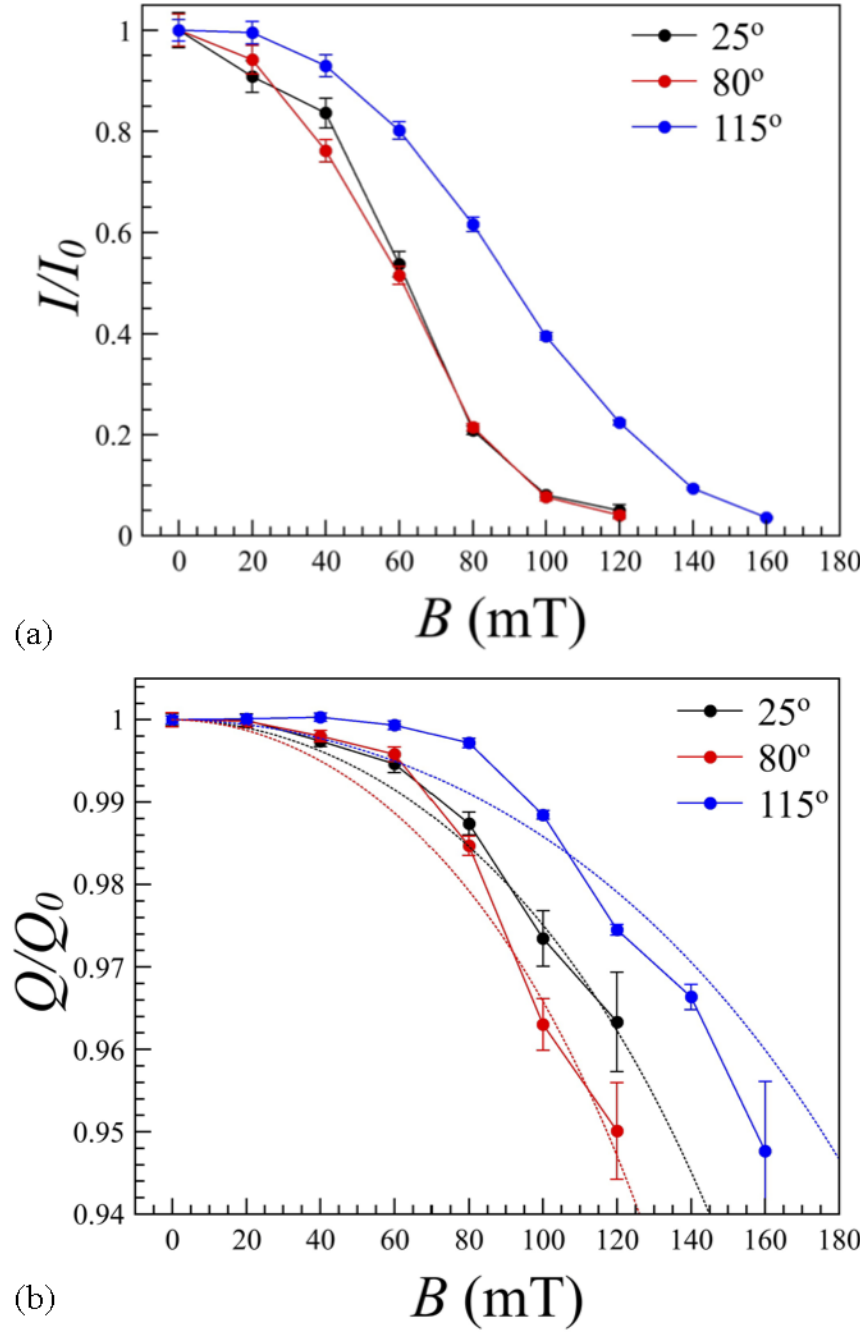

FIG. 4. (a) Dependence of the relative intensity and position (b) of the helical Bragg peak on applied out-of-plane magnetic field for the initial states trained at $\theta=25^{\circ}, 80^{\circ}, 115^{\circ}$. Dotted lines in panel (b) show the fitted $Q$ dependencies.

are oriented parallel to each other and perpendicular to the surface planes. Thus, surface effects do not affect the metastability of the eventual helices. In contrast, when the conical phase is prepared by applying a magnetic field perpendicular to the sample surfaces, then when this field is switched off the helices relax into those with an in-plane $\mathbf{Q}$ oriented along the strain direction due to the surface effects, which are significant in this case [17].

Finally, we discuss the $\theta$ variation in the helical-to-conical transition field $B_{c 1}$ determined from REXS patterns obtained upon out-of-plane field sweeping after an initial in-plane field training [Fig. 4(a)]. The out-of-plane field dependence of the REXS patterns has been measured for the three initial helical states with the propagation vectors $Q$ along $\theta=25^{\circ}, 80^{\circ}$, and $115^{\circ}$. When the out-of-plane field is increased above $B_{c 1}$, the helical state transforms to the conical one with the $q$ vector oriented parallel to the field direction, and therefore its contribution to REXS is not detected in the present experimental geometry. For the case of a strained cubic helimagnet this 
field-induced transition from the in-plane helical to the out-ofplane conical state is mediated by the in-plane chiral soliton lattice (CSL) [17,48,60], which is different from, for example, the $\mathrm{CrNb}_{3} \mathrm{~S}_{6}$ uniaxial helimagnet, where the conical phase is suppressed and a helical to field-polarized phase transition takes place via CSL phase [61].

As evident from the field dependence of the helical peak intensity shown in Fig. 4(a), the transition field $B_{c 1}\left(\theta=25^{\circ}\right) \approx$ $B_{c 1}\left(\theta=80^{\circ}\right) \approx 60 \mathrm{mT}, B_{c 1}\left(\theta=115^{\circ}\right) \approx 90 \mathrm{mT}$ at $I / I_{0}=$ 0.5 . Figure 4(b) shows that the field dependence of $Q / Q_{0}$ also varies with $\theta$. This behavior can be evidently attributed to an easy-plane anisotropy perpendicular to the strain direction $\left(\theta \approx 135^{\circ}\right)$, as introduced in Ref. [17]. It was observed previously in the same sample that formation of a CSL under an applied out-of-plane field takes place when the helical $Q$ vector is oriented perpendicularly to the direction of the strain. Fitting the field dependence of $Q / Q_{0}$ with the sine-Gordon model for a CSL [62] provided the following saturation field values for the soliton modulation depending on the initial state: $B_{\text {sat }}\left(\theta=25^{\circ}\right) \approx 210 \mathrm{mT}, B_{\text {sat }}\left(\theta=80^{\circ}\right) \approx 240 \mathrm{mT}$, $B_{\text {sat }}\left(\theta=115^{\circ}\right) \approx 310 \mathrm{mT}$. Reduction of these critical fields for the spirals tilted from the easy-plane direction agrees well with the theory of simple helix in an external field in the presence of first-order easy-plane anisotropy [62,63]. While the energy of the conical spiral state is equal in all three experimentally measured cases, the energies of initial states are different and dependent on the in-plane angle of $\mathbf{Q}$. The zerofield energy for the $\theta=80^{\circ}$ helix is slightly larger than for the $\theta=25^{\circ}$ one, which in turn is larger than at $\theta=115^{\circ}$, this being simply related to the angle between the easy-plane and corresponding spiral planes. Since the out-of-plane magnetic field should compensate the difference in energy between helicoid and conical phase (see Ref. [17] for details), we arrive to the experimentally observed result: $B_{c 1}\left(\theta=25^{\circ}\right) \approx B_{c 1}(\theta=$ $\left.80^{\circ}\right)<B_{c 1}\left(\theta=115^{\circ}\right)$. Unfortunately, the limited angular range in the present experimental setting did not allow us to directly observe the higher-order Bragg peaks for further characterization of the CSL state. This is thus left as an avenue for future work.

\section{CONCLUSIONS}

From our vector-field resonant small-angle soft x-ray scattering study of an FeGe lamella, we observed the effective reorientation of the helical spin texture along any azimuthal direction under an in-plane training magnetic field. This observation was reproduced by micromagnetic simulations. We have further found that the magnitude of the spiral propagation vector $|\mathbf{Q}|$ changes continuously as a function of the inplane training-field direction with respect to the crystal axes. This is an effect that has not been directly observed before for FeGe, and the angle dependence is accurately described in terms of the higher-order anisotropic term in the exchange interaction. The reasonable estimation of the anisotropic exchange interaction constant might be relevant for further refinement of the ab initio models that currently fail to explain the helical pitch in FeGe [30]. Moreover, a temperaturedependent anisotropic exchange might explain in part the complicated temperature variations of the spiral length in $\beta$-Mn-type alloys $[64,65]$ and chiral molybdenum nitrides [66]. Finally, we note that in FeGe a similar switching of the helical propagation vector by spin transfer torque has been demonstrated, and a memory cell based on this effect has been proposed [67]. The detailed understanding and account of the anisotropic effect given in the present work may be needed to accurately design spin devices based on this concept.

\section{ACKNOWLEDGMENTS}

We thank S.V. Grigoriev for fruitful discussions. Synchrotron measurements were carried out at the VEKMAG instrument at Helmholtz-Zentrum Berlin für Materialien und Energie as a part of the proposal 192-08453 ST. The research leading to this result has been supported by the project CALIPSOplus under the Grant Agreement 730872 from the EU Framework Programme for Research and Innovation HORIZON 2020. V.U., L.Y., and J.S.W. acknowledge support from the SNSF Sinergia CRSII5_171003 NanoSkyrmionics. Contribution to the study by O.U. was funded by the Russian Federation President Grant No. MK-1366.2021.1.2.
[1] S. Mühlbauer, B. Binz, F. Jonietz, C. Pfleiderer, A. Rosch, A. Neubauer, R. Georgii, and P. Böni, Skyrmion lattice in a chiral magnet, Science 323, 915 (2009).

[2] N. Nagaosa and Y. Tokura, Topological properties and dynamics of magnetic skyrmions, Nat. Nanotechnol. 8, 899 (2013).

[3] A. Fert, V. Cros, and J. Sampaio, Skyrmions on the track, Nat. Nanotechnol. 8, 152 (2013).

[4] X. Z. Yu, Y. Onose, N. Kanazawa, J. Park, J. Han, Y. Matsui, N. Nagaosa, and Y. Tokura, Real-space observation of a two-dimensional skyrmion crystal, Nature (London) 465, 901 (2010).

[5] E. Moskvin, S. Grigoriev, V. Dyadkin, H. Eckerlebe, M. Baenitz, M. Schmidt, and H. Wilhelm, Complex Chiral Modulations in FeGe Close to Magnetic Ordering, Phys. Rev. Lett. 110, 077207 (2013).

[6] XZ. Yu, N. Kanazawa, Y. Onose, K. Kimoto, W. Zhang, S. Ishiwata, Y. Matsui, and Y. Tokura, Near room-temperature formation of a skyrmion crystal in thin-films of the helimagnet FeGe, Nat. Mater. 10, 106 (2011).

[7] N. A. Porter, C. S. Spencer, R. C. Temple, C. J. Kinane, T. R. Charlton, S. Langridge, and C. H. Marrows, Manipulation of the spin helix in FeGe thin films and FeGe/fe multilayers, Phys. Rev. B 92, 144402 (2015).

[8] X. Yu, D. Morikawa, T. Yokouchi, K. Shibata, N. Kanazawa, F. Kagawa, T.-h. Arima, and Y. Tokura, Aggregation and collapse dynamics of skyrmions in a non-equilibrium state, Nat. Phys. 14, 832 (2018).

[9] E. Turgut, A. Park, K. Nguyen, A. Moehle, D. A. Muller, and G. D. Fuchs, Chiral magnetic excitations in FeGe films, Phys. Rev. B 95, 134416 (2017).

[10] X. Z. Yu, N. Kanazawa, W. Zhang, T. Nagai, T. Hara, K. Kimoto, Y. Matsui, Y. Onose, and Y. Tokura, Skyrmion flow near room temperature in an ultralow current density, Nat. Commun. 3, 988 (2012). 
[11] K. Shibata, T. Tanigaki, T. Akashi, H. Shinada, K. Harada, K. Niitsu, D. Shindo, N. Kanazawa, Y. Tokura, and T.-h. Arima, Current-driven motion of domain boundaries between skyrmion lattice and helical magnetic structure, Nano Lett. 18, 929 (2018).

[12] X. Yu, D. Morikawa, K. Nakajima, K. Shibata, N. Kanazawa, T.-h. Arima, N. Nagaosa, and Y. Tokura, Motion tracking of 80-nm-size skyrmions upon directional current injections, Sci. Adv. 6, eaaz9744 (2020).

[13] G. Berruto, I. Madan, Y. Murooka, G. M. Vanacore, E. Pomarico, J. Rajeswari, R. Lamb, P. Huang, A. J. Kruchkov, Y. Togawa, T. LaGrange, D. McGrouther, H. M. Ronnow, and F. Carbone, Laser-Induced Skyrmion Writing and Erasing in an Ultrafast Cryo-Lorentz Transmission Electron Microscope, Phys. Rev. Lett. 120, 117201 (2018).

[14] K. Chai, Z.-A. Li, R. Liu, B. Zou, M. Farle, and J. Li, Dynamics of chiral state transitions and relaxations in an FeGe thin plate via in situ lorentz microscopy, Nanoscale 12, 14919 (2020).

[15] K. Shibata, J. Iwasaki, N. Kanazawa, S. Aizawa, T. Tanigaki, M. Shirai, T. Nakajima, M. Kubota, M. Kawasaki, H. Park et al., Large anisotropic deformation of skyrmions in strained crystal, Nat. Nanotechnol. 10, 589 (2015).

[16] N. Kanazawa, J. S. White, H. M. Rønnow, C. D. Dewhurst, Y. Fujishiro, A. Tsukazaki, Y. Kozuka, M. Kawasaki, M. Ichikawa, F. Kagawa, and Y. Tokura, Direct observation of anisotropic magnetic field response of the spin helix in FeGe thin films, Phys. Rev. B 94, 184432 (2016).

[17] V. Ukleev, Y. Yamasaki, O. Utesov, K. Shibata, N. Kanazawa, N. Jaouen, H. Nakao, Y. Tokura, and T.-h. Arima, Metastable solitonic states in the strained itinerant helimagnet FeGe, Phys. Rev. B 102, 014416 (2020).

[18] S. Budhathoki, A. Sapkota, K. M. Law, S. Ranjit, B. Nepal, B. D. Hoskins, A. S. Thind, A. Y. Borisevich, M. E. Jamer, T. J. Anderson, A. D. Koehler, K. D. Hobart, G. M. Stephen, D. Heiman, T. Mewes, R. Mishra, J. C. Gallagher, and A. J. Hauser, Room-temperature skyrmions in strain-engineered FeGe thin films, Phys. Rev. B 101, 220405(R) (2020).

[19] I. Dzyaloshinsky, A thermodynamic theory of "weak" ferromagnetism of antiferromagnetics, J. Phys. Chem. Solids 4, 241 (1958)

[20] T. Moriya, Anisotropic superexchange interaction and weak ferromagnetism, Phys. Rev. 120, 91 (1960).

[21] P. Bak and M. H. Jensen, Theory of helical magnetic structures and phase transitions in mnsi and FeGe, J. Phys. C 13, L881 (1980).

[22] S. V. Maleyev, Cubic magnets with Dzyaloshinskii-Moriya interaction at low temperature, Phys. Rev. B 73, 174402 (2006).

[23] B. Lebech, J. Bernhard, and T. Freltoft, Magnetic structures of cubic FeGe studied by small-angle neutron scattering, J. Phys.: Condens. Matter 1, 6105 (1989).

[24] S. V. Grigoriev, V. A. Dyadkin, D. Menzel, J. Schoenes, Y. O. Chetverikov, A. I. Okorokov, H. Eckerlebe, and S. V. Maleyev, Magnetic structure of $\mathrm{Fe}_{1-x} \mathrm{Co}_{x} \mathrm{Si}$ in a magnetic field studied via small-angle polarized neutron diffraction, Phys. Rev. B 76, 224424 (2007).

[25] S. V. Grigoriev, V. A. Dyadkin, E. V. Moskvin, D. Lamago, T. Wolf, H. Eckerlebe, and S. V. Maleyev, Helical spin structure of $\mathrm{Mn}_{1-y} \mathrm{Fe}_{y} \mathrm{Si}$ under a magnetic field: Small angle neutron diffraction study, Phys. Rev. B 79, 144417 (2009).
[26] S. V. Grigoriev, A. S. Sukhanov, and S. V. Maleyev, From spiral to ferromagnetic structure in B20 compounds: Role of cubic anisotropy, Phys. Rev. B 91, 224429 (2015).

[27] S. V. Grigoriev, S. V. Maleyev, A. I. Okorokov, Y. O. Chetverikov, R. Georgii, P. Böni, D. Lamago, H. Eckerlebe, and K. Pranzas, Critical fluctuations in mnsi near $\mathrm{t}$ c: A polarized neutron scattering study, Phys. Rev. B 72, 134420 (2005).

[28] S. V. Grigoriev, S. V. Maleyev, A. I. Okorokov, Y. O. Chetverikov, and H. Eckerlebe, Field-induced reorientation of the spin helix in MnSi near $\mathrm{T}_{\mathrm{c}}$, Phys. Rev. B 73, 224440 (2006).

[29] X. Wan, Y. Hu, and B. Wang, Exchange-anisotropy-induced intrinsic distortion, structural transition, and rotational transition in skyrmion crystals, Phys. Rev. B 98, 174427 (2018).

[30] S. Grytsiuk, M. Hoffmann, J.-P. Hanke, P. Mavropoulos, Y. Mokrousov, G. Bihlmayer, and S. Blügel, Ab initio analysis of magnetic properties of the prototype B20 chiral magnet FeGe, Phys. Rev. B 100, 214406 (2019).

[31] S. L. Zhang, A. Bauer, H. Berger, C. Pfleiderer, G. Van Der Laan, and T. Hesjedal, Resonant elastic x-ray scattering from the skyrmion lattice in $\mathrm{Cu}_{2} \mathrm{OSeO}_{3}$, Phys. Rev. B 93, 214420 (2016).

[32] S. Zhang, I. Stasinopoulos, T. Lancaster, F. Xiao, A. Bauer, F. Rucker, A. Baker, A. Figueroa, Z. Salman, F. Pratt et al., Roomtemperature helimagnetism in FeGe thin films, Sci. Rep. 7, 123 (2017).

[33] M. J. Stolt, Z.-A. Li, B. Phillips, D. Song, N. Mathur, R. E. Dunin-Borkowski, and S. Jin, Selective chemical vapor deposition growth of cubic FeGe nanowires that support stabilized magnetic skyrmions, Nano Lett. 17, 508 (2017).

[34] A. Chacon, L. Heinen, M. Halder, A. Bauer, W. Simeth, S. Mühlbauer, H. Berger, M. Garst, A. Rosch, and C. Pfleiderer, Observation of two independent skyrmion phases in a chiral magnetic material, Nat. Phys. 14, 936 (2018).

[35] F. Qian, L. J. Bannenberg, H. Wilhelm, G. Chaboussant, L. M. Debeer-Schmitt, M. P. Schmidt, A. Aqeel, T. T. Palstra, E. Brück, A. J. Lefering et al., New magnetic phase of the chiral skyrmion material $\mathrm{Cu}_{2} \mathrm{OSeO}_{3}$, Sci. Adv. 4, eaat7323 (2018).

[36] L. J. Bannenberg, H. Wilhelm, R. Cubitt, A. Labh, M. P. Schmidt, E. Lelièvre-Berna, C. Pappas, M. Mostovoy, and A. O. Leonov, Multiple low-temperature skyrmionic states in a bulk chiral magnet, npj Quantum Mater. 4, 11 (2019).

[37] Y. Yamasaki, D. Morikawa, T. Honda, H. Nakao, Y. Murakami, N. Kanazawa, M. Kawasaki, T. Arima, and Y. Tokura, Dynamical process of skyrmion-helical magnetic transformation of the chiral-lattice magnet FeGe probed by small-angle resonant soft x-ray scattering, Phys. Rev. B 92, 220421(R) (2015).

[38] V. Ukleev, Y. Yamasaki, D. Morikawa, N. Kanazawa, Y. Okamura, H. Nakao, Y. Tokura et al., Coherent resonant soft $\mathrm{X}$-ray scattering study of magnetic textures in FeGe, Quantum Beam Sci. 2, 3 (2018).

[39] V. Ukleev, Y. Yamasaki, D. Morikawa, K. Karube, K. Shibata, Y. Tokunaga, Y. Okamura, K. Amemiya, M. Valvidares, H. Nakao et al., Element-specific soft x-ray spectroscopy, scattering, and imaging studies of the skyrmion-hosting compound $\mathrm{Co}_{8} \mathrm{Zn}_{8} \mathrm{Mn}_{4}$, Phys. Rev. B 99, 144408 (2019).

[40] D. M. Burn, S. L. Zhang, S. Wang, H. F. Du, G. Van Der Laan, and T. Hesjedal, Helical magnetic ordering in thin FeGe membranes, Phys. Rev. B 100, 184403 (2019). 
[41] D. M. Burn, S. Wang, W. Wang, G. van der Laan, S. Zhang, H. $\mathrm{Du}$, and T. Hesjedal, Field and temperature dependence of the skyrmion lattice phase in chiral magnet membranes, Phys. Rev. B 101, 014446 (2020).

[42] M. N. Wilson, M. T. Birch, A. Štefančič, A. C. TwitchettHarrison, G. Balakrishnan, T. J. Hicken, R. Fan, P. Steadman, and P. D. Hatton, Stability and metastability of skyrmions in thin lamellae of $\mathrm{Cu}_{2} \mathrm{OSeO}_{3}$, Phys. Rev. Res. 2, 013096 (2020).

[43] C. Tabata, Y. Yamasaki, Y. Yokoyama, R. Takagi, T. Honda, Y. Kousaka, J. Akimitsu, and H. Nakao, Observation of chiral magnetic soliton lattice state in $\mathrm{CrNb}_{3} \mathrm{~S}_{6}$ by coherent soft x-ray diffraction imaging, JPS Conf. Proc. 30, 011194 (2020).

[44] K. Chen, D. Lott, A. Philippi-Kobs, M. Weigand, C. Luo, and F. Radu, Observation of compact ferrimagnetic skyrmions in $\mathrm{DyCo}_{3}$ film, Nanoscale 12, 18137 (2020).

[45] N. Kanazawa, Magnetic and transport properties in B20-type germanides, in Charge and Heat Transport Phenomena in Electronic and Spin Structures in B20-type Compounds (Springer Japan, Tokyo, 2015) pp. 29-44.

[46] F. Büttner, M. Schneider, C. M. Günther, C. Vaz, B. Lägel, D. Berger, S. Selve, M. Kläui, and S. Eisebitt, Automatable sample fabrication process for pump-probe $\mathrm{x}$-ray holographic imaging, Opt. Express 21, 30563 (2013).

[47] Y. Okamura, Y. Yamasaki, D. Morikawa, T. Honda, V. Ukleev, H. Nakao, Y. Murakami, K. Shibata, F. Kagawa, S. Seki et al., Directional electric-field induced transformation from skyrmion lattice to distinct helices in multiferroic $\mathrm{Cu}_{2} \mathrm{OSeO}_{3}$, Phys. Rev. B 95, 184411 (2017).

[48] Y. Okamura, Y. Yamasaki, D. Morikawa, T. Honda, V. Ukleev, H. Nakao, Y. Murakami, K. Shibata, F. Kagawa, S. Seki et al., Emergence and magnetic-field variation of chiral-soliton lattice and skyrmion lattice in the strained helimagnet $\mathrm{Cu}_{2} \mathrm{OSeO}_{3}$, Phys. Rev. B 96, 174417 (2017).

[49] T. Noll and F. Radu, The mechanics of the VEKMAG experiment, in Proceedings, 9th Mechanical Engineering Design of Synchrotron Radiation Equipment and Instrumentation (MEDSI 2016) (JACoW Publishing, Geneva, Switzerland, 2016), p. 370 .

[50] A. Vansteenkiste, J. Leliaert, M. Dvornik, M. Helsen, F. GarciaSanchez, and B. Van Waeyenberge, The design and verification of MuMax3, AIP Adv. 4, 107133 (2014).

[51] R. Takagi, D. Morikawa, K. Karube, N. Kanazawa, K. Shibata, G. Tatara, Y. Tokunaga, T. Arima, Y. Taguchi, Y. Tokura, and S. Seki, Spin-wave spectroscopy of the DzyaloshinskiiMoriya interaction in room-temperature chiral magnets hosting skyrmions, Phys. Rev. B 95, 220406(R) (2017).

[52] P. Schoenherr, J. Müller, L. Köhler, A. Rosch, N. Kanazawa, Y. Tokura, M. Garst, and D. Meier, Topological domain walls in helimagnets, Nat. Phys. 14, 465 (2018).

[53] A. O. Leonov, Y. Togawa, T. L. Monchesky, A. N. Bogdanov, J. Kishine, Y. Kousaka, M. Miyagawa, T. Koyama, J. Akimitsu, T. Koyama, K. Harada, S. Mori, D. McGrouther, R. Lamb, M. Krajnak, S. McVitie, R. L. Stamps, and K. Inoue, Chiral Surface Twists and Skyrmion Stability in Nanolayers of Cubic Helimagnets, Phys. Rev. Lett. 117, 087202 (2016).
[54] F. Zheng, F. N. Rybakov, A. B. Borisov, D. Song, S. Wang, Z.-A. Li, H. Du, N. S. Kiselev, J. Caron, A. Kovács et al., Experimental observation of chiral magnetic bobbers in B20type FeGe, Nat. Nanotechnol. 13, 451 (2018).

[55] S. M. Vlasov, V. M. Uzdin, and A. O. Leonov, Skyrmion flop transition and congregation of mutually orthogonal skyrmions in cubic helimagnets, J. Phys.: Condens. Matter 32, 185801 (2020).

[56] T. Ma, A. K. Sharma, R. Saha, A. K. Srivastava, P. Werner, P. Vir, V. Kumar, C. Felser, and S. S. P. Parkin, Tunable magnetic antiskyrmion size and helical period from nanometers to micrometers in a $D_{2 d}$ heusler compound, Adv. Mater. 32, 2002043 (2020).

[57] D. A. Gilbert, A. J. Grutter, P. M. Neves, G.-J. Shu, G. Zimanyi, B. B. Maranville, F.-C. Chou, K. Krycka, N. P. Butch, S. Huang et al., Precipitating ordered skyrmion lattices from helical spaghetti and granular powders, Phys. Rev. Mater. 3, 014408 (2019).

[58] Y. A. Izyumov and V. Laptev, Diffraction of neutron by incommensurate magnetic-structures, Sov. Phys. JETP 85, 2185 (1983).

[59] O. I. Utesov and A. V. Syromyatnikov, Spiral plane flops in frustrated helimagnets in external magnetic field, Phys. Rev. B 98, 184406 (2018).

[60] T. Nakajima, V. Ukleev, K. Ohishi, H. Oike, F. Kagawa, S.-i. Seki, K. Kakurai, Y. Tokura, and T.-h. Arima, Uniaxialstress effects on helimagnetic orders and skyrmion lattice in $\mathrm{Cu}_{2} \mathrm{OSeO}_{3}$, J. Phys. Soc. Jpn. 87, 094709 (2018).

[61] Y. Togawa, T. Koyama, K. Takayanagi, S. Mori, Y. Kousaka, J. Akimitsu, S. Nishihara, K. Inoue, A. Ovchinnikov, and J.-i. Kishine, Chiral Magnetic Soliton Lattice on a Chiral Helimagnet, Phys. Rev. Lett. 108, 107202 (2012).

[62] Y. A. Izyumov and V. Laptev, Neutron diffraction by incommensurate magnetic strcutures, Sov. Phys. JETP 58, 1267 (1983).

[63] G. W. Paterson, A. A. Tereshchenko, S. Nakayama, Y. Kousaka, J. Kishine, S. McVitie, A. S. Ovchinnikov, I. Proskurin, and Y. Togawa, Tensile deformations of the magnetic chiral soliton lattice probed by lorentz transmission electron microscopy, Phys. Rev. B 101, 184424 (2020).

[64] K. Karube, J. S. White, N. Reynolds, J. L. Gavilano, H. Oike, A. Kikkawa, F. Kagawa, Y. Tokunaga, H. M. Rønnow, Y. Tokura et al., Robust metastable skyrmions and their triangular-square lattice structural transition in a high-temperature chiral magnet, Nat. Mater. 15, 1237 (2016).

[65] K. Karube, J. S. White, V. Ukleev, C. D. Dewhurst, R. Cubitt, A. Kikkawa, Y. Tokunaga, H. M. Rønnow, Y. Tokura, and Y. Taguchi, Metastable skyrmion lattices governed by magnetic disorder and anisotropy in $\beta$-mn-type chiral magnets, Phys. Rev. B 102, 064408 (2020).

[66] A. S. Sukhanov, A. Heinemann, L. Kautzsch, J. D. Bocarsly, S. D. Wilson, C. Felser, and D. S. Inosov, Robust metastable skyrmions with tunable size in the chiral magnet $\mathrm{FePtMo}_{3} \mathrm{~N}$, Phys. Rev. B 102, 140409(R) (2020).

[67] J. Masell, X. Yu, N. Kanazawa, Y. Tokura, and N. Nagaosa, Combing the helical phase of chiral magnets with electric currents, Phys. Rev. B 102, 180402(R) (2020). 